\title{
Valuing Demand Response Controllability via Chance Constrained Programming
}

\author{
Kenneth Bruninx, Member, IEEE, Yury Dvorkin, Member, IEEE, \\ Erik Delarue, Member, IEEE, William D'haeseleer, Member, IEEE, and Daniel S. Kirschen, Fellow, IEEE
}

\begin{abstract}
Controllable loads can modify their electricity consumption in response to signals from a system operator, providing some of the flexibility needed to compensate for the stochasticity of electricity generated from renewable energy sources (RES) and other loads. However, unlike traditional flexibility providers, e.g. conventional generators and energy storage systems, demand response (DR) resources are not fully controlled by the system operator and their availability is limited by user-defined comfort constraints. This paper describes a deterministic unit commitment model with probabilistic reserve constraints that optimizes day-ahead power plant scheduling in the presence of stochastic RES-based electricity generation and DR resources that are only partially controllable, in this case residential electric heating systems. This model is used to evaluate the operating cost savings that can be attained with these DR resources on a model inspired by the Belgian power system.
\end{abstract}

Index Terms-Demand response, limited controllability, uncertainty, unit commitment

\section{INTRODUCTION}

$\mathbf{S}$ YSTEM operators (SO) can use the flexibility provided by residential demand response (DR) resources, e.g. thermostatically controlled and deferrable loads, to compensate for the stochasticity of electricity generated from renewable energy sources (RES) and other loads [1]-[3]. The wholesystem value of this flexibility, which depends on the particular DR technology considered [4], has been demonstrated as a form of arbitrage [3], [5], [6] and for several types of regulation services [1], [2], [7]-[10].

The whole-system value of DR with thermostatically controlled loads has been assessed in [3], [5], [6], [8]-[10] . Mathieu et al. [3] investigate the value of DR-based arbitrage in Californias intraday energy market by leveraging the thermal inertia of thermostatically controlled loads. Operating cost savings from the perspective of an aggregator amount from USD 2 to USD 37 per thermostatically controlled load per year. Patteeuw et al. [5] and Arteconi et al. [6] employ a model similar to the one used in this study, but make the following

K. Bruninx, E. Delarue and W. D'haeseleer are with the University of Leuven (KU Leuven) Energy Institute, TME Branch (Energy Conversion), Celestijnenlaan 300A box 2421, B-3001 Leuven, Belgium and EnergyVille, Thor Park 8310, B-3600 Genk. K. Bruninx is also with VITO, the Flemish Institute for Technological Research, Boeretang 200, B-2400 Mol.

Y. Dvorkin is with Department of Electrical and Computer Engineering, New York University, New York, NY 11201, USA.

D. S. Kirschen is with Department of Electrical Engineering, University of Washington, Seattle, WA 98195-2500, USA.

Corresponding authors: Erik Delarue, erik.delarue@kuleuven.be and Daniel S. Kirschen, kirschen@uw.edu.

Manuscript received January 2, 2017; revised April 11, 2017; accepted June $18,2017$. simplifying assumptions: (i) the power system is represented using a merit order model that neglects intertemporal constraints, (ii) the stochastic nature of the RES-based generation is neglected and (iii) DR-based arbitrage is assumed perfectly controllable. The case study in [5] shows that the $\mathrm{CO}_{2}$ abatement cost associated with the electrification of heating can be drastically reduced if thermostatically controlled loads are used for energy arbitrage. Arteconi et al. [6] determine the operating cost savings as a function of the number of DR loads in a future Belgian power system. Annual operating cost savings range from $€ 112$ to $€ 35$ per heat pump. If these DR loads are also used to mitigate peak loads, their value increases by $€ 163$ to $€ 315$ in deferred investments in peak capacity. Li et al. [8] and Zhang et al. [9] study the value of DR-based reserves with unknown availabilities using chance constraints. In contrast to our approach, Zhang et al. [9] use a simplified, single period optimal power flow (OPF) formulation, which does not consider DR-based arbitrage and does not account for the activation cost of reserves. Similarly, Li et al. [8] consider a multi-period AC OPF problem for a 30-bus IEEE test system, but neglect the activation cost of reserves. O'Connell et al. [10] evaluate the potential of flexible supermarket refrigeration loads to offer regulating power using an economic dispatch model. The DR resource is represented using saturation curves and assumed perfectly controllable. The cost of providing and activating DR-based reserves is fixed and disconnected from the ex-ante energy cost to ensure the availability of this regulating power.

However, the residential DR models considered in some of these studies simplify the complex interactions between the supply and demand sides, which may lead to erroneous estimates of the value of the DR resources [11]. Although some researchers study the impact of an uncertain availability of DR resources [8], [9], these tools customarily assume that SOs have perfect control over the DR resources, i.e. that they have the ability to externally adjust the state of these loads [2], [3]. In practice, DR from residential customers is unlikely to match the expectations of the SO exactly [12] because of inaccuracies in the weather forecast and in the load model (e.g. behavior of the occupants, limited information on the state, constraints and dynamics of the loads [13], [14].) This paper proposes an extended deterministic unit commitment model with endogenous, probabilistic reserve sizing and allocation (DUC-PR) [15] that optimizes dayahead scheduling decisions in the presence of RES-based generation with limited predictability and residential DR with limited controllability. Numerical experiments with this model 
demonstrate that achieving operating cost savings attained with residential DR, while minimizing customer grievances and lost utility [1], [16], requires a better characterization and modeling of its controllability. The focus is on short-term load shifting (arbitrage) and the provision of regulation services by space and water heating systems, leveraging the inherent thermal storage in the buildings and hot water storage tanks. We consider the provision of secondary and tertiary reserve (frequency restoration and replacement reserves). In the rest of this paper these will be referred to as reserves and regulation (services) interchangeably. This paper makes the following contributions:

1) It describes a multi-period, deterministic UC formulation considering probabilistic reserve constraints. This formulation simultaneously accounts for inter-temporal constraints on all physical assets and computes an expectation of the recourse cost. Using this formulation makes it possible to make day-ahead decisions with respect to the intra-day balancing cost and to estimate the ability of residential DR loads to provide temporal energy arbitrage and regulation services, assuming direct load control by the $\mathrm{SO}^{1}$.

2) It uses chance constraints to model the limited controllability of the DR resources. These chance constraints are analytically reformulated as second-order conic (SOC) constraints [19], [20] to enhance the computational tractability of the proposed DUC-PR formulation. This representation is demonstrated to account for the limited controllability of the DR loads.

3) Using a model inspired by the Belgian power system, it quantifies the operating cost savings obtained with residential DR resources with limited controllability. This case study provides numerical estimates based on realistic data.

Regulators, policy makers and power system operators may use the method described below to assess the whole-system value of DR, which may inform a detailed cost-benefit analysis of the deployment of DR infrastructure. Power system operators may integrate this framework with their daily routines to account for the limited controllability of DR resources and their own risk-attitude.

\section{DUC-PR WITH RESIDENTIAL DR}

The proposed UC model extends the DUC-PR model described in [15] by integrating residential DR resources providing load shifting and ancillary services [11]. This model is therefore referred to as an integrated (DUC-PR) model, in accord with the terminology suggested in [11]. We adopt the perspective of a vertically-integrated utility or SO, which bears all the operating costs associated with supplying the demand. Using this model, the SO schedules the available conventional and RES-based generation, the energy storage

\footnotetext{
${ }^{1}$ Assuming a direct load control approach and a SO-perspective makes it possible to study the theoretical maximum of the whole-system value attained with residential DR resources [17]. Interested readers are referred to [18] for a comparison of incentive-based (direct load control, curtailable load) and pricebased (real-time pricing, time-of-use pricing, peak pricing) control approaches.
}

(ES) systems and the residential DR resources to minimize the system operating cost. We assume that the system operator is responsible for all scheduling decisions and transactions. Wind power forecasts are the only source of uncertainty considered in this model. All resources are modelled as perfectly controllable, except the DR loads in Sections II-B and III-D. Physical constraints imposed on the residential DR schedule ensure that the user-defined comfort levels are met under all possible realizations of the uncertain quantities.

\section{A. Integrated DUC-PR with residential $D R$}

The objection function of the DUC-PR model ${ }^{2}$ is [11]:

$$
\begin{aligned}
& \min \sum_{j}\left(\sum_{i}\left(c_{i j}^{\mathrm{SU}}+c_{i j}^{\mathrm{F}}+c_{i j}^{\mathrm{E}}+c_{i j}^{\mathrm{R}}\right)+T P \cdot \operatorname{VOLL}\left(\phi_{j} \quad(1)\right.\right. \\
& \left.\left.+\sum_{l} P_{j l}^{+} \cdot \phi_{j l}^{+}\right)+\sum_{i} \sum_{l}\left(P_{j l}^{+}\left(c_{i j l}^{\mathrm{SR}+}+c_{i j l}^{\mathrm{NSR}+}\right)+P_{j l}^{-} \cdot c_{i j l}^{\mathrm{SR}-}\right)\right)
\end{aligned}
$$

where the first term accounts for the the start-up $\left(c_{i j}^{\mathrm{SU}} \geq 0\right)$, fuel $\left(c_{i j}^{\mathrm{F}} \geq 0\right), \mathrm{CO}_{2}$-emission $\left(c_{i j}^{\mathrm{E}} \geq 0\right)$ and ramping $\left(c_{i j}^{\mathrm{R}} \geq 0\right)$ costs of each conventional generator $i$ (set $I$ ) at time step $j$ (set $J$, length of each time step $T P$ ) under forecast conditions. The fuel, $\mathrm{CO}_{2}$-emission and ramping costs depend on the dispatch of each conventional generator. The second term represents the cost of load shedding $\left(\phi_{j} \geq 0\right)$, which only occurs if the scheduled resources are not sufficient to meet the load under forecast conditions, and the penalty cost of relaxing upward reserve requirements (Eq. (11), $\phi_{j l}^{+} \geq 0$ ) at reserve level $l$ (set $L$ ) with the associated activation probability $P_{j l}^{+}$[15]. Load shedding and relaxing the upward reserve requirement are penalized at the value of lost load (VOLL). The third term represents the expected activation cost of reserves computed based on the activation probability of upward $\left(P_{j l}^{+}\right)$and downward $\left(P_{j l}^{-}\right)$reserve level $l$ and the deployment cost of upward $\left(c_{i j l}^{\mathrm{SR}+}\right)$ and downward spinning $\left(c_{i j l}^{\mathrm{SR}-}\right)$ and upward non-spinning $\left(c_{i j l}^{\mathrm{NSR}+}\right)$ reserves. Variable $c_{i j l}^{\mathrm{SR}+} \geq 0$ includes the additional fuel and $\mathrm{CO}_{2}$-emission costs associated with deploying upward spinning reserves, while variable $c_{i j l}^{\mathrm{NSR}+} \geq 0$ also includes the start-up cost of these fast-starting units. Variable $c_{i j l}^{\mathrm{SR}-} \leq 0$ includes possible fuel and $\mathrm{CO}_{2}$-emission cost savings associated with deploying downward reserves to accommodate increases in renewable generation [15]. Since we take the system perspective, the energy storage facility and DR loads are operated at no explicit cost to the system operator. However, charging/discharging of an energy storage facility results in energy losses due to round-trip inefficiencies. Similarly, shifting the electric heating demand may lead to increased thermal losses and a higher overall energy consumption (Section III-B, Fig. 2c). These losses are taken into account when the least-cost day-ahead UC schedule is determined. Similarly, reserves provided by energy storage systems, residential DR and RES-based generation are scheduled at no explicit cost. However, ensuring their availability may increase their respective scheduled consumption, and thus their operating cost, under forecast conditions (see below (DR) and [21] (ES)).

\footnotetext{
${ }^{2}$ In the presented formulation, upper and lower case letters denote parameters and decision variables, respectively.
} 
This optimization problem is constrained as follows:

1) Power balance constraint:

$\forall j: \quad D_{j}+d_{j}^{\mathrm{H}}-\phi_{j}=\sum_{i} g_{i j}+G_{j}^{\mathrm{F}}-\chi_{j}+\sum_{r} g_{r j}^{\mathrm{ES}}$,

Equation (2) ensures that the inflexible demand $\left(D_{j}\right)$ and the flexible electricity demand of residential electric heating systems $\left(d_{j}^{\mathrm{H}} \geq 0\right)$ are met by the power output of conventional generators $\left(g_{i j} \geq 0\right)$, the forecast wind power output $\left(G_{j}^{\mathrm{F}}\right)$, which can be curtailed $\left(0 \leq \chi_{j} \leq G_{j}^{\mathrm{F}}\right)$, and the net injections from the ES systems $\left(g_{r j}^{\mathrm{ES}}\right.$, free variable), indexed by $r$ (set $R$ ). If the available generation resources are insufficient to meet the total demand $\left(D_{j}+d_{j}^{\mathrm{H}}\right)$, load shedding $\left(\phi_{j}\right)$ occurs.

2) Residential DR constraints: The flexible demand $d_{j}^{\mathrm{H}}$ in Eq. (2) is modeled as follows:

$$
\begin{aligned}
\forall j: & d_{j}^{\mathrm{H}}=\sum_{h} N B_{h} \cdot\left(p_{h j}^{\mathrm{HP}}+p_{h j}^{\mathrm{A}}\right) \\
\forall h, \forall j: & p_{h j}^{\mathrm{HP}}=p_{h j}^{\mathrm{HP}, \mathrm{SH}}+p_{h j}^{\mathrm{HP}, \mathrm{HW}} \leq \overline{P_{h}^{\mathrm{HP}}} \\
\forall h, \forall j: & p_{\mathrm{hj}}^{\mathrm{A}}=p_{h j}^{\mathrm{A}, \mathrm{SH}}+p_{h j}^{\mathrm{A}, \mathrm{HW}} \leq \overline{P_{h}^{\mathrm{A}}},
\end{aligned}
$$

where $N B_{h}$ denotes the number of buildings of each type $h$ (set $H$ ) providing DR. Each building is equipped with a heat pump (HP) and an auxiliary heater (A) to provide space heating $(\mathrm{SH})$ and hot water (HW). Equation (3) aggregates the electrical power required by heat pumps $\left(p_{h j}^{\mathrm{HP}}\right)$ and auxiliary heaters $\left(p_{h j}^{\mathrm{A}}\right)$, which are limited to their nameplate capacity $\overline{P_{h}^{\mathrm{HP}}}$ and $\overline{P_{h}^{\mathrm{A}}}$ in (4) and (5). We distinguish between the electrical power required for space heating $\left(p_{\mathrm{hij}}^{\mathrm{HP}, \mathrm{SH}} \geq 0\right.$ and $\left.p_{h j}^{\mathrm{A}, \mathrm{SH}} \geq 0\right)$ and hot water production $\left(p_{h j}^{\mathrm{HP}, \mathrm{HW}} \geq 0\right.$ and $p_{h j}^{\mathrm{A}, \mathrm{HW}} \geq 0$ ).

The electrical power consumed by the heat pump and the auxiliary heater is related to the required thermal power for space heating $\dot{q}_{h j}^{\mathrm{SH}} \geq 0$ using the coefficient of performance $\left(C O P_{h}^{\mathrm{SH}}\right)$ of each heat pump $h$ :

$\forall h, \forall j: \quad \dot{q}_{h j}^{\mathrm{SH}}=C O P_{h}^{\mathrm{SH}} \cdot p_{h j}^{\mathrm{HP}, \mathrm{SH}}+p_{h j}^{\mathrm{A}, \mathrm{SH}}$,

The thermal behavior of each building and its heat emission system is simulated by a linear state-space model $\left(A_{h p}, B_{h p}\right)$ :

$\forall h, \forall p, \forall j: \quad t_{h p j}^{\mathrm{SH}}=A_{h p} \cdot t_{h p, j-1}^{\mathrm{SH}}+B_{h p} \cdot \dot{q}_{\mathrm{hj}}^{\mathrm{SH}}+E_{h p j}^{\mathrm{SH}}$

$\forall h, \forall p, \forall j: \quad \underline{T_{h p j}^{\mathrm{SH}}} \leq t_{h p j}^{\mathrm{SH}} \leq \overline{T_{h p j}^{\mathrm{SH}}}$.

Equation (7) describes the evolution of the temperature $\left(t_{h p j}^{\mathrm{SH}} \geq\right.$ 0 ) in each temperature state $p$ (set $P$ ), which is related to the thermal power supplied to each building $\left(\dot{q}_{h j}^{\mathrm{SH}}\right)$ and the thermal losses, internal and solar gains $E_{h p j}^{\mathrm{SH}}$. Equation (8) enforces the user-defined comfort constraints imposed on the indoor air temperature $\left(T_{h p j}^{\mathrm{SH}}, \overline{T_{h p j}^{\mathrm{SH}}}\right)$. Similarly, the electric power required to produce hot water is computed as follows [22]:

$$
\begin{aligned}
\forall h, \forall j: \quad t_{h j}^{\mathrm{HW}} & =t_{h, j-1}^{\mathrm{HW}}-\frac{T P \cdot G_{h}}{C_{h}} \cdot\left(t_{h j}^{\mathrm{HW}}-T^{\mathrm{E}}\right) \\
& +\frac{T P}{C_{h}} \cdot\left(C O P_{h}^{\mathrm{HW}} \cdot p_{h j}^{\mathrm{HP}, \mathrm{HW}}+p_{h j}^{\mathrm{A}, \mathrm{HW}}-\dot{Q}_{h j}^{\mathrm{D}}\right)
\end{aligned}
$$$$
\forall h, \forall j: \quad T_{h j}^{\mathrm{HW}} \leq t_{h j}^{\mathrm{HW}} \leq \overline{T_{h}^{\mathrm{HP}}} .
$$

Equation (9) describes the evolution of the temperature of the water $t_{h j}^{\mathrm{HW}}$ in the hot water storage tank, which depends on the thermal conductance of the storage tank $G_{h}$, the temperature of its surroundings $T^{\mathrm{E}}$, the thermal power supplied to the hot water storage tank and the thermal capacity of the storage tank and its contents $C_{h}$. $\dot{Q}_{h j}^{\mathrm{D}}$ represents the withdrawal of thermal power from the hot water storage tank due to hot water consumption, which follows a predefined profile. Equation (10) ensures the user-defined availability of hot water at temperatures between $T_{h j}^{\mathrm{HW}}$ and $\overline{T_{h j}^{\mathrm{HP}}}$. As shown in [23] and [5], these linear state-space models approximate the nonlinear dynamics of residential buildings with heat pumps with sufficient accuracy for system-level studies.

3) Reserve constraints: The upward and downward reserve requirements are divided into $L$ levels, each with a specific activation probability $\left(P_{j l}^{+}, P_{j l}^{-}\right)$, as explained in [15]. The reserve requirements $\left(D_{j l}^{+}, D_{j l}^{-}\right)$for each reserve level $l$ read:

$$
\begin{aligned}
\forall j, \forall l: D_{j l}^{+} & =\sum_{i}\left(r_{i j l}^{+}+n s r_{i j l}^{+}\right)+\sum_{r} r_{r j l}^{\mathrm{ES}+}+\chi_{j l}^{+} \\
& +\phi_{j l}^{+}+\sum_{h} N B_{h} \cdot r_{h j l}^{\mathrm{H}+} \\
\forall j, \forall l: D_{j l}^{-} & =\sum_{i} r_{i j l}^{-}+\sum_{r} r_{r j l}^{\mathrm{ES}-}+\chi_{j l}^{-} \\
& +\sum_{h} N B_{h} \cdot r_{h j l}^{\mathrm{H}-} .
\end{aligned}
$$

Conventional spinning $\left(r_{i j l}^{+}, r_{i j l}^{-} \geq 0\right)$ and non-spinning reserves $\left(n s r_{i j l}^{+} \geq 0\right)$ are limited to the available headroom and ramping capacity of generator $i$ at each time step $j$. The ES-based reserves $\left(r_{r j l}^{\mathrm{ES}+}, r_{r j l}^{\mathrm{ES}-} \geq 0\right)$ are restricted by the power and energy storage capacity of the ES systems, as well as a set of constraints related to their scheduled output under forecast conditions [15], [21]. Upward RESbased reserves $\left(\chi_{j l}^{+} \geq 0\right)$ are limited to the foreseen curtailed RES-based generation under forecast conditions. Downward RES-based reserves require additional curtailment of RESbased electricity generation. The reserves provided by the residential DR resources $\left(r_{h j l}^{\mathrm{H}+}, r_{h j l}^{\mathrm{H}-} \geq 0\right)$ are limited by the scheduled consumption under forecast conditions and capacity of the electric heating systems:

$$
\begin{aligned}
\forall h, \forall j: \quad \sum_{l} r_{h j l}^{\mathrm{H}+} & =r_{h j}^{\mathrm{HP}, \mathrm{SH}+}+r_{h j}^{\mathrm{HP}, \mathrm{HW}+} \\
& +r_{h j}^{\mathrm{A}, \mathrm{SH}+}+r_{h j}^{\mathrm{A}, \mathrm{HW}+} \\
\forall h, \forall j: \quad \sum_{l} r_{h j l}^{\mathrm{H}-} & =r_{h j}^{\mathrm{HP}, \mathrm{SH}-}+r_{h j}^{\mathrm{HP}, \mathrm{HW}-} \\
& +r_{h j}^{\mathrm{A}, \mathrm{SH}-}+r_{h j}^{\mathrm{A}, \mathrm{HW}-}
\end{aligned}
$$

$\forall h, \forall j: \quad p_{h j}^{\mathrm{HP}}+r_{h j}^{\mathrm{HP}, \mathrm{SH}-}+r_{h j}^{\mathrm{HP}, \mathrm{HW}-} \leq \overline{P_{h}^{\mathrm{HP}}}$

$\forall h, \forall j: p_{h j}^{\mathrm{A}}+r_{h j}^{\mathrm{A}, \mathrm{SH}-}+r_{h j}^{\mathrm{A}, \mathrm{HW}-} \leq \overline{P_{h}^{\mathrm{A}}}$

$\forall h, \forall j: r_{h j}^{\mathrm{HP}, \mathrm{SH}+} \leq p_{h j}^{\mathrm{HP}, \mathrm{SH}}$

$\forall h, \forall j: \quad r_{h j}^{\mathrm{A}, \mathrm{SH}+} \leq p_{h j}^{\mathrm{A}, \mathrm{SH}}$

$\forall h, \forall j: \quad r_{h j}^{\mathrm{HP}, \mathrm{HW}+} \leq p_{h j}^{\mathrm{HP}, \mathrm{HW}}$

$\forall h, \forall j: \quad r_{h j}^{\mathrm{A}, \mathrm{HW}+} \leq p_{h j}^{\mathrm{A}, \mathrm{HW}}$

The DR-based reserves $r_{i j l}^{\mathrm{H}+} \geq 0$ and $r_{i j l}^{\mathrm{H}-} \geq 0$ may be scheduled if and only if their deployment does not result in a violation of the comfort constraints (Eq. (8) and (10)) during real-time dispatch (see Section III). This is achieved by enforcing the following constraints, e.g. for the upward 
DR-based reserves related to space heating:

$$
\begin{aligned}
& \forall h, \forall j: \quad \dot{q}_{h j}^{\mathrm{SH}+}=p_{h j}^{\mathrm{A}, \mathrm{SH}}-r_{h j}^{\mathrm{A}, \mathrm{SH}+} \\
&+C O P_{\mathrm{h}}^{\mathrm{HP}, \mathrm{SH}} \cdot\left(p_{h j}^{\mathrm{HP}, \mathrm{SH}}-r_{h j}^{\mathrm{HP}, \mathrm{SH}+}\right) \\
& \forall h, \forall p, \forall j: \quad t_{h p j}^{\mathrm{SH}+}=A_{h p} \cdot t_{h p, j-1}^{\mathrm{SH}+}+B_{h p} \cdot \dot{q}_{\mathrm{hj}}^{\mathrm{SH}+}+E_{h p j}^{\mathrm{SH}} \\
& \forall h, \forall p, \forall j: \quad \underline{T_{h p j}^{\mathrm{SH}}-\Delta T \leq t_{\mathrm{hpj}}^{\mathrm{SH}+}}
\end{aligned}
$$

If upward DR-based reserves related to space heating are activated, this results in less thermal power supplied to the associated buildings $\left(\dot{q}_{h j}^{\mathrm{SH}+} \geq 0\right)$, which in turn results in lower indoor air temperatures $\left(t_{h p j}^{\mathrm{SH}+} \geq 0\right)$. By limiting this temperature to the comfort range (Eq. (23)), one ensures that even in the worst-case scenario, i.e. when all upward reserves are deployed, the resulting temperature $t_{\mathrm{hpj}}^{\mathrm{SH}+}$ remains above $T_{h p j}^{\mathrm{SH}}-\Delta T$. Similar constraints are enforced to ensure that the real-time activation of downward DR-based reserves $\left(r_{h j}^{\mathrm{A}, \mathrm{SH}-}, r_{h j}^{\mathrm{HP}, \mathrm{SH}-}\right)$ does not lead to temperatures greater than $\overline{T_{h p j}^{\mathrm{SH}}}+\Delta T:$

$$
\begin{aligned}
& \forall h, \forall j: \quad \dot{q}_{h j}^{\mathrm{SH}-}=p_{h j}^{\mathrm{A}, \mathrm{SH}}+r_{h j}^{\mathrm{A}, \mathrm{SH}-} \\
& +C O P_{\mathrm{h}}^{\mathrm{HP}, \mathrm{SH}} \cdot\left(p_{h j}^{\mathrm{HP}, \mathrm{SH}}+r_{h j}^{\mathrm{HP}, \mathrm{SH}-}\right) \\
& \forall h, \forall p, \forall j: \quad t_{h p j}^{\mathrm{SH}-}=A_{h p} \cdot t_{h p, j-1}^{\mathrm{SH}-}+B_{h p} \cdot \dot{q}_{\mathrm{hj}}^{\mathrm{SH}-}+E_{h p j}^{\mathrm{SH}} \\
& \forall h, \forall p, \forall j: \quad t_{h p j}^{\mathrm{SH}-} \leq \overline{T_{h p j}^{\mathrm{SH}}}+\Delta T .
\end{aligned}
$$

Although constraints (21)-(26) result in a conservative scheduling of the DR-based regulation services, they do ensure that the scheduled reserves can be deployed without loss of comfort. If one would like to reduce this conservatism, one could (1) loosen the temperature bounds imposed in the worstcase evaluations using the parameter $\Delta T$ (Eq. (23) and Eq. (26), which is the approach we will use in Section III-C) and/or (2) allow some discomfort, i.e. violations of the temperature band, penalized in the objective function at a so-called cost of discomfort [24]. In the Monte Carlo dispatch simulations (see Section III), the original temperature bounds are imposed on the optimization problem and the thermal discomfort is minimized by penalizing it at a high cost in the objective function. Load shedding is not allowed in these dispatch simulations. All reserve inadequacies must therefore be solved by violating the thermal comfort requirements. Unlike parameters $\overline{T_{h p j}^{\mathrm{SH}}}$ and $T_{h p j}^{\mathrm{SH}}$ that are set by individual occupants and may vary based on their geographical location, parameter $\Delta T$ represents the comfort violation and is uniform for the entire system. Using a uniform $\Delta T$-value makes it possible for the system operator to treat all consumers indiscriminately and to assess the benefits attained by violating thermal comfort constraints conservatively.

Similarly, worst-case comfort constraints on the temperature of the hot water are enforced by duplicating Eq. (9)-(10) for a worst-case deployment scenario. If one neglects the impact of a change in the temperature of the water on the heat losses, one can approximate these worst-case evaluations using the following two constraints:

$$
\begin{aligned}
\forall h, \forall j: \quad t_{\mathrm{h}, \mathrm{j}}^{\mathrm{HW}}-\sum_{j^{*}=1}^{j} \frac{T P}{C_{h}} \cdot\left(C O P_{\mathrm{hj}^{*}}^{\mathrm{HP}, \mathrm{HW}} \cdot r_{\mathrm{h}}^{\mathrm{HP}, \mathrm{HW}+}\right. \\
\left.\quad+r_{\mathrm{hj}^{*}}^{\mathrm{A}, \mathrm{HW}+}\right) \geq \underline{T_{\mathrm{hj}}^{\mathrm{HW}}} \\
\forall h, \forall j: \quad t_{\mathrm{hj}}^{\mathrm{HW}}+\sum_{j^{*}=1}^{j} \frac{T P}{C_{h}} \cdot\left(C O P_{\mathrm{h}}^{\mathrm{HP}, \mathrm{HW}} \cdot r_{\mathrm{hj}^{*}, \mathrm{HW}-}^{\mathrm{HP}}\right. \\
\left.+r_{\mathrm{hj}^{*}}^{\mathrm{A}, \mathrm{HW}-}\right) \leq \overline{T_{\mathrm{h}}^{\mathrm{HP}}}
\end{aligned}
$$

This approximation is acceptable because $G_{h}$ is typically a small number. Moreover, as the thermal losses to the environment are proportional to the temperature difference $t_{\mathrm{hj}}^{\mathrm{HW}}-T^{\mathrm{E}}$ (see Eq. (9)), we (1) overestimate the impact of deploying upward reserves and (2) underestimate the impact of deploying downward reserves on the hot water temperature. The lower temperature bound $T_{\mathrm{hj}}^{\mathrm{HW}}$ will thus be respected in all cases and all scheduled upward reserves can be deployed in real time without loss of comfort. The deployment of downward reserves may be limited due to the supply temperature limits of the heat pump $\left(\overline{T_{\mathrm{h}}^{\mathrm{HP}}}\right)$, which may result in an increase in curtailment of RES-based electricity generation during dispatch (see Section III). Violations of the comfort constraints w.r.t. the availability of hot water are not allowed.

4) Other UC constraints: The minimum and maximum power output limits, ramp rate constraints, minimum up and down time requirements, start-up and shut-down trajectories of conventional generators are enforced as described in [15]. Similarly, ES systems operations are constrained by their rated energy storage and power capacity as discussed in [21].

\section{B. Controllability of the residential DR}

The DUC-PR model of Section II-A assumes that all DR resources behave exactly as scheduled by the SO. However, residential DR resources do not necessarily behave as expected [13]. We therefore replace the deterministic variables $d_{j}^{\mathrm{H}}, r_{h j l}^{\mathrm{H}+}$ and $r_{h j l}^{\mathrm{H}-}$ by stochastic variables, indicated with a tilde (e.g. $\left.\widetilde{d_{j}^{\mathrm{H}}}\right)$. Constraint (2) is reformulated as a chance constraint:

$$
\begin{gathered}
\operatorname{Pr}\left(\forall j: D_{j}+\widetilde{d_{j}^{\mathrm{H}}}-\phi_{j} \leq \sum_{i} g_{i j}+G_{j}^{\mathrm{F}}-\chi_{j}\right. \\
\left.+\sum_{r} g_{r j}^{\mathrm{ES}}\right) \geq 1-\epsilon,
\end{gathered}
$$

$\epsilon$ is a small number, ensuring that the expression between brackets in Eq. (29) holds in $(1-\epsilon) \cdot 100 \%$ of all possible realizations of the uncertain quantities.

We analyzed the difference between the expected and actual demand of a set of controllable heat pumps, based on data published by Patteeuw et al. [25]. This analysis reveals a weak correlation between this difference and the expected demand and suggests that stochastic variables (e.g. $\widetilde{d_{j}^{\mathrm{H}}}$ ) can be characterized by a proportional $\left(\delta^{\mathrm{P}}\right)$ and a non-proportional $\left(\delta^{\mathrm{NP}}\right)$ component:

$\forall j: \quad \widetilde{d_{j}^{\mathrm{H}}}=\left(1+\delta^{\mathrm{P}}\right) \cdot d_{j}^{\mathrm{H}}+\delta^{\mathrm{NP}}$.

In this paper, $\delta^{\mathrm{P}}$ and $\delta^{\mathrm{NP}}$ are assumed to follow a Normal distribution, i.e. $\delta^{\mathrm{P}} \sim N\left(\mu^{\mathrm{P}},\left(\sigma^{\mathrm{P}}\right)^{2}\right)$ and $\delta^{\mathrm{NP}} \sim$ 
$N\left(\mu^{\mathrm{NP}},\left(\sigma^{\mathrm{NP}}\right)^{2}\right)$. Given these assumptions, chance constraint (29) can be analytically recast as the following second order conic (SOC) constraint [19]:

$$
\begin{aligned}
& \forall j: D_{j}+\left(1+\mu_{j}^{\mathrm{P}}\right) \cdot d_{j}^{\mathrm{H}}+\mu_{j}^{\mathrm{NP}}+\Phi^{-1}(1-\epsilon) \cdot\left(t+\sigma^{\mathrm{NP}}\right) \\
& \quad=\sum_{i} g_{i j}+G_{j}^{\mathrm{F}}-\chi_{j}+\sum_{r} g_{r j}^{\mathrm{ES}}+\phi_{j} \\
& t^{2} \geq \sum_{j}\left(\sigma_{j}^{\mathrm{P}} \cdot d_{j}^{\mathrm{H}}\right)^{2}
\end{aligned}
$$

where $t$ is an auxiliary decision variable. Similarly, the reserve constraints with stochastic variables $r_{h j l}^{\mathrm{H},+}$ and $r_{h j l}^{\mathrm{H},-}$ can be represented by SOC constraints [26]. The resulting formulation is a mixed integer quadratically constrained problem (MIQCP) that can be solved with existing solvers (e.g. CPLEX [19]). Note that other approaches can be used for solving problems containing chance constraints in the form given by Eq. (29), see [27], [28] for further details. The advantage of using the analytical SOC reformulation in the context of this paper is that it does not require scenario sampling and preserves computational resources for solving an NP-hard DUC-PR model.

\section{CASE Study}

We study the system value of DR from residential electric heating systems using a model inspired by the Belgian power system, documented in [26]. Wind energy is assumed to cover $50 \%$ of the annual energy demand. The transmission grid and interconnections with neighboring countries are neglected, as in [15], [21], [26]. All considered buildings are thoroughly insulated, leading to similar DR potentials across building types [5]. The number of buildings is set to $\sum_{h} N B_{h}=10^{6}$ and the building portfolio is represented by an average 2030 low-energy building described in [6], [26]. The temperature bounds $\left(T_{h p j}^{\mathrm{SH}}, \overline{T_{h p j}^{\mathrm{SH}}}, T_{h j}^{\mathrm{HW}}\right)$ aim to represent a possible occupant behavior and can be generated using the method in [29] and aggregated as explained in [22].

The resulting UC schedules are evaluated using Monte Carlo (MC) simulations, as in [21], on a set of 500 wind power scenarios per day generated as described in [26], [30]. For each MC trial, the operation of the power plants, the ES systems and the loads participating in DR are optimized assuming perfect foresight on the available wind power. If the DR resource is imperfectly controllable, the result of each dispatch simulation is re-evaluated on a new set of scenarios representing the DR load. These scenarios are generated as random disturbances of the optimized DR-load profile linked to each wind power scenario. The temporal resolution of all models is 15 minutes.

For the numerical analysis below, four representative weeks were selected based on the estimated operating cost savings in each week. To obtain these estimates, we solve the model above for a full year, but treat the wind power forecast as perfect. No reserve constraints are imposed and the resulting schedule is not re-evaluated in a dispatch simulation. Following the method of Arteconi et al. [6], we calculate the weekly value of DR-based arbitrage as the difference between (1) the total operating cost when the DR loads are perfectly controllable and (2) the total operating cost of the system when the DR loads are non-responsive and only minimize their own energy use. The week in which DR yields the highest (week 7, representing $12 \%$ of the year), lowest (week 15 , representing $32 \%$ of the year) and closest-to-the-average operating cost savings (week 9, representing 30\% of the year) are selected in the heating season. Additionally, one week outside of the heating season is selected (week 25, representing $26 \%$ of the year).

All models are implemented in GAMS v.24.2 using CPLEX v.12.5. Simulations are run on a $2.8 \mathrm{GHz}$ machine with 20 cores and $64 \mathrm{~GB}$ of RAM with an optimality gap of $0.5 \%$. Median computation times are 5.7 minutes (no DR), 10 minutes (perfectly controllable DR providing energy arbitrage) and 26 minutes (perfectly controllable DR providing energy arbitrage and reserve provision). The solutions of these problems are considered as starting values to solve the model considering chance constraint (29), which leads to median computation times of 10 minutes for the problem considering limitedly controllable DR providing energy arbitrage.

The value of perfectly controllable DR-based arbitrage and regulation services is analyzed in Section III-A for one representative day and in Section III-B for four representative weeks. The system value of thermal discomfort is quantified Section III-C. The impact of the limited controllability of DR is assessed in Section III-D.

\section{A. Residential DR and reserve scheduling}

On the sixth day of week 7 , wind energy is capable of covering approximately $42 \%$ of the total electricity demand, of which $15 \%$ is related to space heating and domestic hot water production. Because the demand ${ }^{3}$ and wind power are poorly correlated, the residual demand ${ }^{4}$ exhibits a significant variability (Fig. 1a). This variability affects not only the scheduling of units to meet the demand under forecast conditions, but also the availability of conventional capacity to meet the upward reserve requirements (Fig. 1b).

Introducing perfectly controllable DR-based arbitrage strongly reduces the variability in the residual demand profile (Fig. 1a). The heating demand is shifted to hours of lower consumption, hence lower electricity costs, and 'valley filling' occurs. This results not only in a more cost-effective UC schedule, but also in a more efficient use of the resources needed to meet the reserve requirements. Less spinning reserves (SR), more non-spinning reserves (NSR), more RESbased reserves and less reserve relaxation $\left(\phi^{+}\right)$are scheduled ('DR-arbitrage' - Fig. 1c). The expected operating cost (i.e. as calculated using MC ED simulations) decreases by $16 \%$ and the expected curtailment drops by $40 \%$. The expected energy not served decreases from 1.5 MWh to $0 \mathrm{MWh}$.

Leveraging the DR from electric heating to provide costeffective regulation services (still assuming perfect control-

\footnotetext{
${ }^{3}$ The demand of the electric heating systems that are not taking part in DR ('No DR'), is fixed to the demand profile that results from the minimization of the energy needed to meet the thermal comfort constraints for each building ('minimum energy use' demand profile), without regard for the constraints or operating costs on the supply side, using the same demand side model as in the integrated model.

${ }^{4}$ The residual demand is calculated as the total demand minus the wind power forecast. This is the load profile to be covered by the conventional power plants.
} 


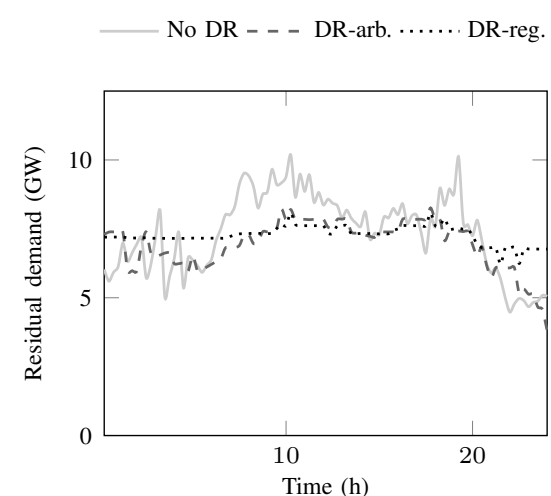

(a) Residual demand

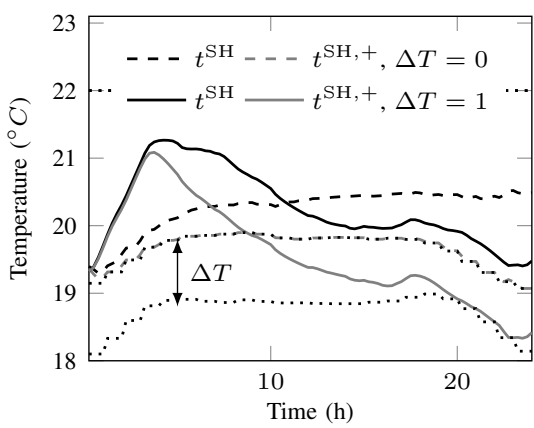

(d) Day-zone temperature (single house)

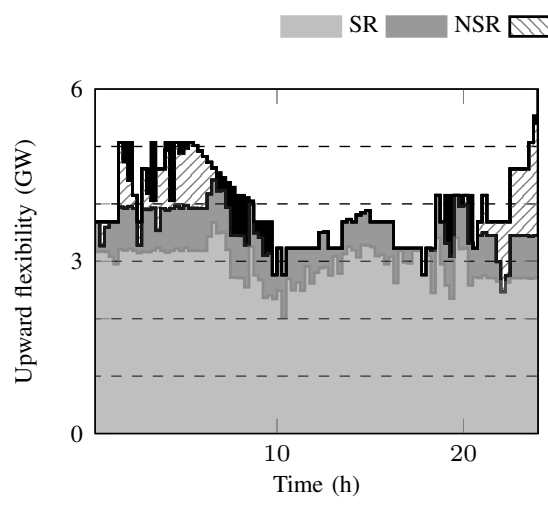

(b) Upward reserves - no DR

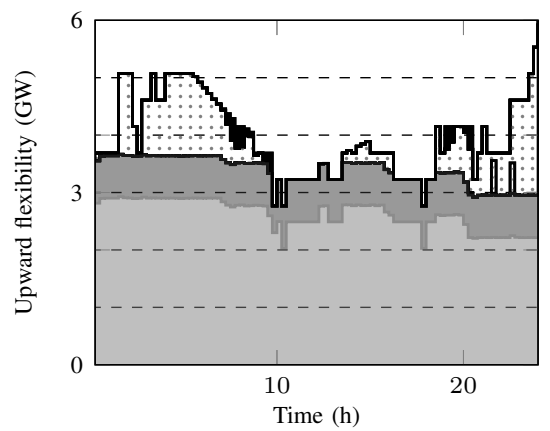

(e) Upward reserves - DR-regulation $(\Delta T=0)$

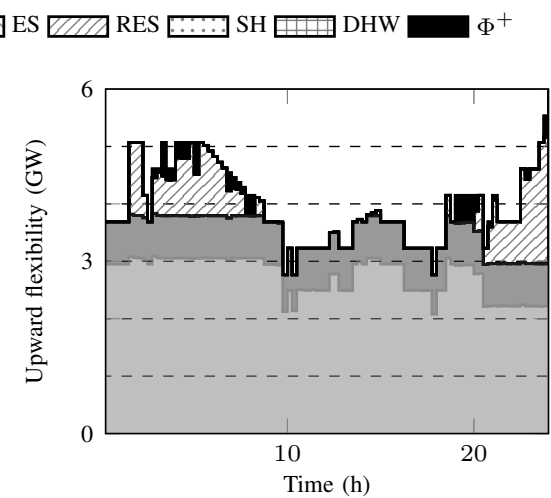

(c) Upward reserves - DR-arbitrage

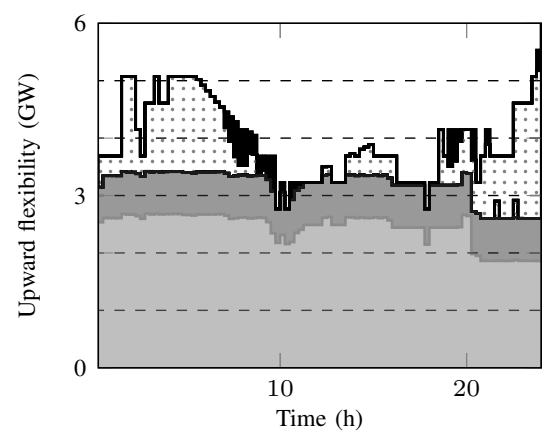

(f) Upward reserves - DR-regulation $\left(\Delta T=1^{\circ} \mathrm{C}\right)$

Fig. 1. Introducing perfectly controllable DR reduces the variability in the residual demand (Fig. 1a), which allows for a more cost-effective UC schedule and reserve procurement (1c). Reserves provided by conventional generation are separated in spinning (SR) and non-spinning reserves (NSR). Energy storage (ES) and RES-based reserves (RES) may further fullfill the demand for reserves. Reserve shedding $\phi^{+}$is scheduled in extreme cases. DR-based reserves (associated with space heating ( $\mathrm{SH}$ ) or domestic hot water production (DHW)) further increase the cost-effectiveness of the UC schedule (Fig. 1e), without violating the thermal comfort constraints of the reserve providers (Fig. 1d) unless the worst-case comfort constraints are relaxed (Fig. 1f).

lability) further decreases the expected operating cost ('DRregulation' - Fig. 1e). Fig. 1a shows the resulting residual demand profile if no thermal discomfort is allowed $(\Delta T=0)$. By increasing the heating demand during periods where curtailment of renewable generation would occur, the temperature in certain buildings increases above the lower temperature bound (dotted line, Fig. 1d). This allows DR to provide upward regulation services (Fig. 1e) without violating the comfort constraints in real-time (dashed grey line, Fig. 1d). The amount of spinning reserves (SR) is slightly reduced, curtailment is no longer needed to meet the reserve requirements and no reserve shedding $\left(\phi^{+}\right)$is scheduled. On this particular day, the expected operating cost decreases by approximately $3 \%$ compared to the 'DR-arbitrage only'-case and 19\% compared to the 'no DR'-case. Curtailment volumes drop by $33 \%$ compared to the 'DR-arbitrage only'-case and 60\% compared to the 'no DR'-case. The reliability is unaffected compared to the 'DR-arbitrage only'-case.

Relaxing the worst-case comfort constraints using $\Delta T$ (Eq. (26) and Fig. 1d, dotted black line), further increases the amount of reserves provided by DR from electric heating (Fig. 1f). This reduces the amount of spinning reserves needed to meet the reserve requirement, but activation of these DRbased reserves no longer guarantees thermal comfort (Fig. 1d). If one does not account for any monetary compensation to the consumers for this thermal discomfort, operating cost savings on this particular day are approximately $0.2 \mathrm{M} €$ or $0.2 € /$ household. This benefit should however be weighted against the thermal discomfort ${ }^{5}$ experienced by consumers providing DR. In this case, this amounts to - on average $0.1 \mathrm{Kh} / \mathrm{hh}$. Recall that load shedding was not allowed during this dispatch simulation (Section II).

\section{B. System value of DR arbitrage and regulation services}

In our four-week analysis, we consider three UC strategies. In the case 'SR' only spinning reserves may be scheduled, while in the 'SR \& NSR' case non-spinning reserves are also available to meet the reserve requirements. In the case ' $S R$, NSR \& ES', spinning, non-spinning and ES-based reserves are available. For each of these cases, we calculate the expected total operating cost $(\mathrm{E}[\mathrm{TOC}])$, the expected wind utilization factor (E[WUF]), the resulting total demand (E[Load]) and the share of electrical energy generated from non-renewable resources (1-E[WS]) for three levels of demand responsiveness. In the reference case ('Ref.'), the load is not responsive at all and the electricity demand of the electric heating systems is fixed to a 'minimum energy use' profile. In the second level, the DR-capable heating systems are only used for arbitrage

\footnotetext{
${ }^{5}$ Thermal discomfort is defined here as the equivalent number of hours, averaged over all households, during which the thermal comfort constraints are violated by $1 \mathrm{~K}$, expressed in $\mathrm{Kh} / \mathrm{hh}$ (Kelvin hour per household).
} 
('Arb.'). For the third level, both arbitrage and regulation services can be procured from the demand side ('Reg.'). We will assume (1) the load that participated in DR is perfectly controllable and (2) thermal comfort is guaranteed if DR-based regulation services are scheduled $(\Delta T=0)$.

Significant cost savings are to be expected from DRbased arbitrage and regulation services (Fig. 2). On average, the operating cost decreases by $6 \%$ when considering DRbased arbitrage ('Arb.', Fig. 2a). An additional one percent decrease can be realized when the load also provides regulation services ('Reg.', Fig. 2a). The reliability of the resulting UC schedules is unaffected as load shedding is estimated at less than $0.0004 \%$ of the total load. Remarkably, the value of DR-based arbitrage and regulation services remains unaffected when other flexibility providers, such as non-spinning reserves ('NSR') and ES-based reserves are available. The presence of these flexibility providers, in particular non-spinning reserves, decreases the operating cost by $4 \%$ on average. During the heating season, the decrease in operating cost as a result of DR-arbitrage varies between 5\% (week 15) and 10\% (week 7). The additional operating cost decrease due to DR-regulation services varies between $1 \%$ (week 7 and 9) and 2\% (week 15). Outside the heating season (week 25), the operating cost decrease resulting from DR-based arbitrage and regulation is less than $2 \%$ due to the significantly lower fraction of the load that is available for DR as no space heating is required. Allowing non-spinning reserves and ES-based reserves yields an expected cost saving of $11 \%$ outside the heating season.

The main driver of these cost reductions is an increased utilization of the available wind power (Fig. 2b) and a more efficient scheduling and dispatching of the conventional power plants. On average, the WUF increases from 74.9\%-77.5\% ('No DR') to $82.1 \%-84.2 \%$ ('Arb.') to $83.4 \%-85.3 \%$ ('Reg.'). This increase in the WUF is the result of (i) shifting demand to periods of excess wind power generation and (ii) increasing the demand to increase the indoor temperature in order to allow the heating systems to provide upward reserves (see Section III-A). However, this does increase the total demand as a result of increased thermal losses (Fig. 2c). The average increase in total demand amounts to $2.5 \%$ ('ES', 'Arb.') to $3.2 \%$ ('SR', 'Reg.'). The availability of non-spinning and ESbased reserves limits the increase in demand, as less excess wind power is available to be absorbed by the heating systems (Fig. 2b). On the contrary, the consideration of DR-based reserves typically increases the total demand due to the higher indoor temperatures required to provide upward reserves. As a result, the share of non-renewable energy sources in the fuel mix (Fig. 2d) does not decrease as fast as the WUF increases. On average, $67.7 \%$ to $66.2 \%$ of the demand would be satisfied with electricity generated from non-RES in the absence of DR. This drops to $65.6 \%-64.4 \%$ and $65.1 \%-63.9 \%$ when considering DR-based arbitrage and regulation respectively.

\section{System value of thermal discomfort}

Figure 3 shows how the expected total operating cost varies when $\Delta T$ varies from 0 (reference case) to $2 \mathrm{~K}$. The cost of thermal discomfort, i.e. the equivalent number of hours

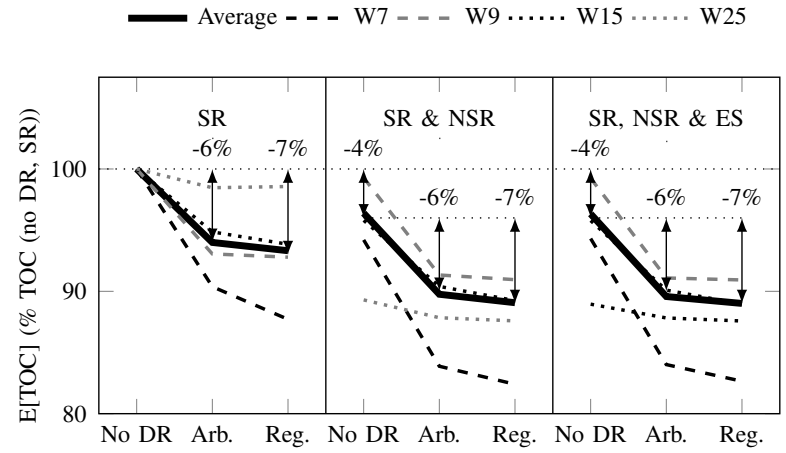

(a) Expected total operating cost (TOC), normalized w.r.t. the expected operating cost under the assumption of only spinning reserves (SR) and no DR.

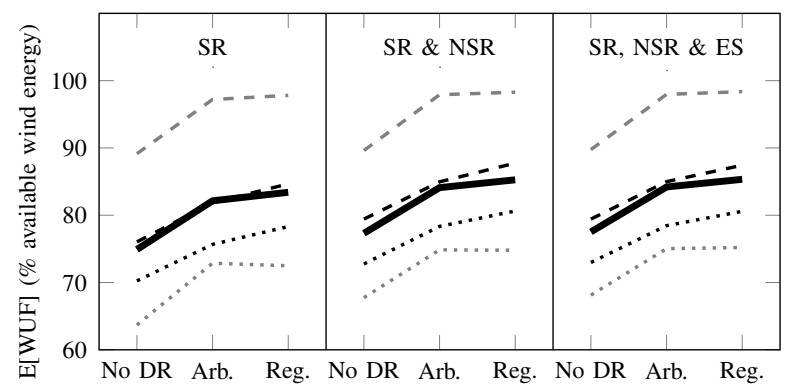

(b) Wind Utilization Factor (WUF)

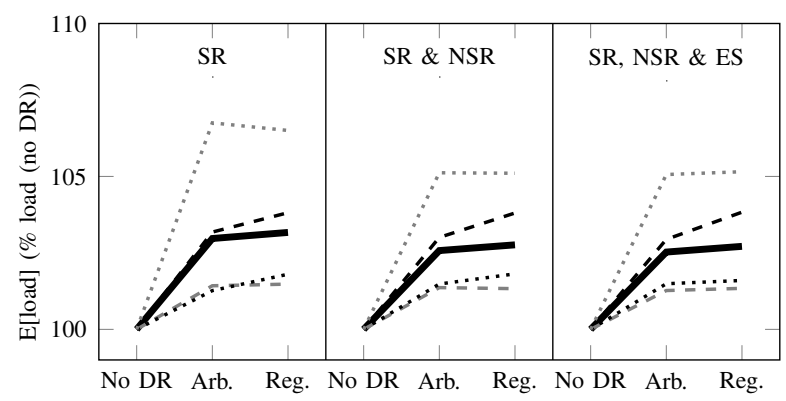

(c) Total demand, normalized w.r.t to the demand if the heating systems do not provide DR.

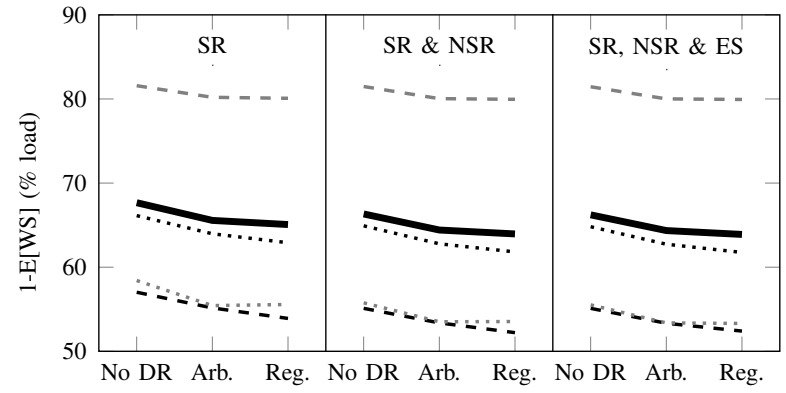

(d) Share of the demand covered by non-renewables sources

Fig. 2. Leveraging residential DR leads to significant operating cost savings (Fig. 2a), even in the presence of other cost-effective flexibility providers (spinning (SR), non-spinning (NSR) and ES-based reserves). This is predominantly caused by an increased wind utilization (Fig. 2b), as a result of DR-based arbitrage. The resulting thermal losses lead to an increase in demand (Fig. 2c), which limits the decrease in the proportion of electricity generated from non-renewable energy sources (1-E[WS], with WS being the share of wind energy in the total demand) (Fig. 2d). 
that the thermal comfort bounds are violated by $1 \mathrm{~K}$, is not included. These operating cost reductions, attainable by allowing thermal discomfort, must therefore be interpreted as upper bounds of the system value of thermal discomfort, not corrected for the monetary compensation that occupants who experience thermal discomfort would require. If only spinning and DR-based reserves can be procured, violating the thermal comfort constraints under 'worst-case conditions' during UC scheduling reduces the expected total operating cost by $2 \%$ to $3 \%$ on average. The inclusion of non-spinning and ES-based reserves decreases the expected operating cost by 3 to $4 \%$ (average values) w.r.t. the situation where only spinning and DR-based reserves $(\Delta T=0 \mathrm{~K})$ can be scheduled. Allowing thermal discomfort under 'worst-case conditions' during UC scheduling yields a total expected operating cost reduction of $5 \%(\Delta T=1 \mathrm{~K})$ to $6 \%(\Delta T=2 \mathrm{~K})$. Note however that the differences between the three considered weeks increase. In week 9, non-spinning reserves result in an expected operating cost decrease of $2 \% . \Delta T$-values of $1 \mathrm{~K}$ and $2 \mathrm{~K}$ yield respectively an additional $1 \%$ and $2 \%$ decrease. In contrast, the inclusion of non-spinning reserves in week 7 , in which the introduction of DR-based arbitrage and regulation services had the largest impact (Fig. 2a), allows for a $7 \%$ decrease in expected operating cost $(\Delta T=0 \mathrm{~K})$. If thermal discomfort under 'worst-case conditions' is allowed during UC scheduling, this results in an additional decrease in operating cost of up to $7 \%(\Delta T=2 \mathrm{~K})$.

The resulting operating cost decrease (Fig. 3a) should be weighted against the expected thermal discomfort experienced by the owners of heating system who participate in DR. Figure $3 b$ shows the average thermal discomfort per household, as a function of the $\Delta T$-value considered during UC scheduling. Thermal discomfort values range between 1.3 and $3 \mathrm{Kh} / \mathrm{hh} /$ week $(\Delta T=1 \mathrm{~K})$ and 2.9 and $4.8 \mathrm{Kh} / \mathrm{hh} /$ week $(\Delta T$ $=2 \mathrm{~K}$ ). In week 7 , for which we observed significant operating cost reductions as a result of non-zero $\Delta T$-values (Fig. 3a), the associated thermal discomfort varies between 2.3 and 12 $\mathrm{Kh} / \mathrm{hh} /$ week (spinning and DR-based reserves), 11.3 and 15.6 $\mathrm{Kh} / \mathrm{hh} /$ week (spinning, non-spinning and DR-based reserves) and 11.4 and $19.3 \mathrm{Kh} / \mathrm{hh} /$ week (spinning, non-spinning, ESbased and DR-based reserves). In week 7, thermal discomfort is also observed in the results corresponding to a UC schedule obtained with $\Delta T$ equal to zero, as load shedding was not allowed in these simulations and insufficient reserves were procured to meet the demand. Redistributing the operating cost savings (Fig. 3b) across all DR-consumers results in an estimate of the possible average compensation for thermal discomfort of 0.13 to $0.42 € / \mathrm{Kh}$. The highest compensation is observed in results that show little thermal discomfort $(\Delta T$ $=1 \mathrm{~K}$ ) and were obtained considering only spinning and DRbased reserves. In week 7 , in which relaxing the worst-case comfort constraint on the DR-based reserves results in the largest operating cost savings and thermal discomfort, this value would vary between 0.06 to $0.42 € / \mathrm{Kh}$.

\section{Effect of limited controllability of DR}

To illustrate the impact that limited controllability may have on the system value of DR loads, we focus on a particular

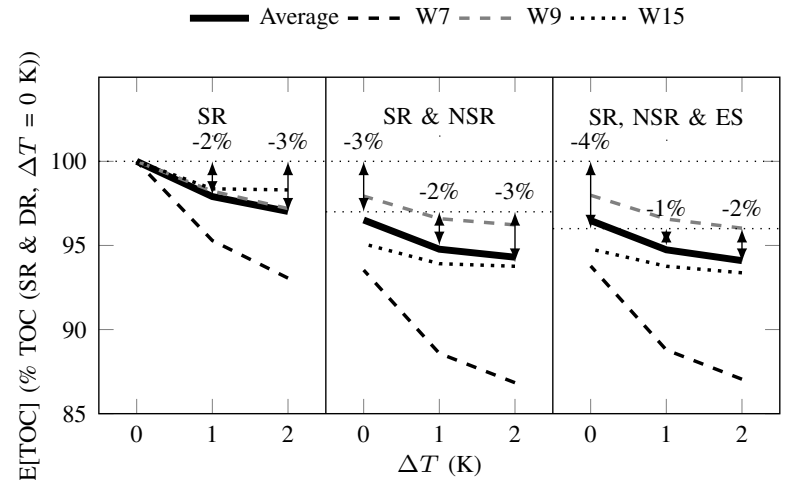

(a) Expected total operating cost (TOC), normalized w.r.t. expected total operating cost obtained if only spinning and DR-based reserves are available, but thermal discomfort is not allowed $(\Delta T=0 \mathrm{~K})$.

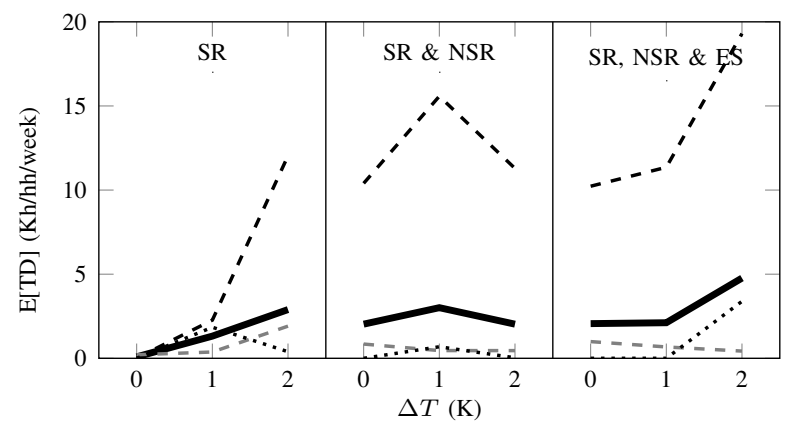

(b) Expected thermal discomfort (TD) per household per week, based on a dedicated MC ED evaluation of the UC schedules obtained with the corresponding $\Delta T$-value.

Fig. 3. Allowing thermal discomfort under 'worst-case conditions', limited by parameter $\Delta T$, during UC scheduling yields significant reductions in expected operating cost (Fig. 3a), but results in thermal discomfort during real-time dispatch (Fig. 3b). Note that the penalties associated with the thermal discomfort are not included in the expected total operating cost (TOC).

setting for week 7, where DR achieved the highest operating cost savings. We assume DR is only used for arbitrage and the controllability of the DR loads can be characterized by a non-proportional error term $\left(\delta^{\mathrm{NP}} \sim N\left(\mu^{\mathrm{NP}},\left(\sigma^{\mathrm{NP}}\right)^{2}\right)\right)$ with a zero mean $\left(\mu^{\mathrm{NP}}=0 \mathrm{MW}\right)$ and three $\sigma^{\mathrm{NP}}$-values $(50 \mathrm{MW}$, $100 \mathrm{MW}$ and $250 \mathrm{MW}$ ). These values are to be compared with the average electric heating demand during week 7 , which is approximately 2,000 MW. The SO schedules the power plants, energy storage systems, spinning, non-spinning and ES-based reserves day-ahead, considering the limited controllability of DR-based arbitrage through chance constraints (29).

Not considering the limited controllability of DR loads in the MC ED trials, we obtain an estimate of the operating cost increase the SO would incur by scheduling the electricity generation system accounting for the limited controllability of the loads (dashed lines, Fig. 4a). If $\epsilon$, which is the probability that the UC schedule will be inadequate to meet the load, equals 0.01 , the corresponding UC schedule is capable of meeting the load in $99 \%(1-\epsilon)$ of all realizations of the DR events. This will require scheduling more units and a lower utilization of the available wind power (Fig. 4b), which increases the expected operating cost (dashed lines, Fig. 4a). In contrast, if $\epsilon=0.5$, the SO does not account for the limited controllability of the DR loads, given the symmetry of the Gaussian distribution and its zero mean. Hence, the 


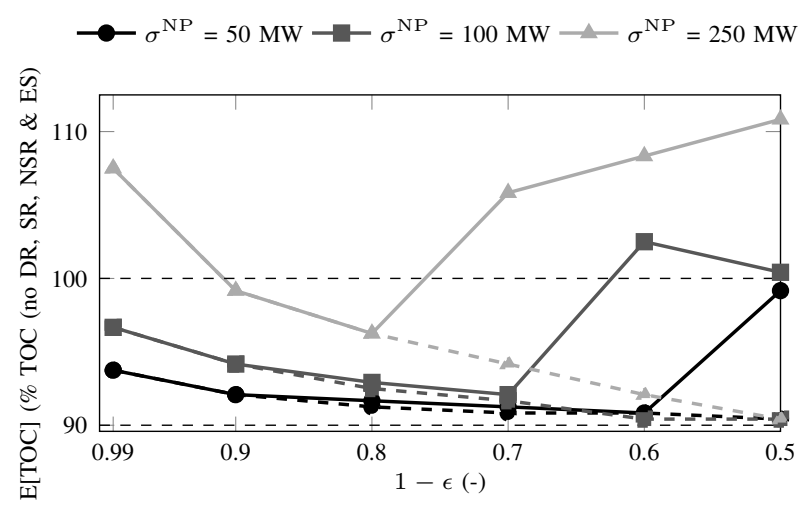

(a) Total operating cost (TOC)

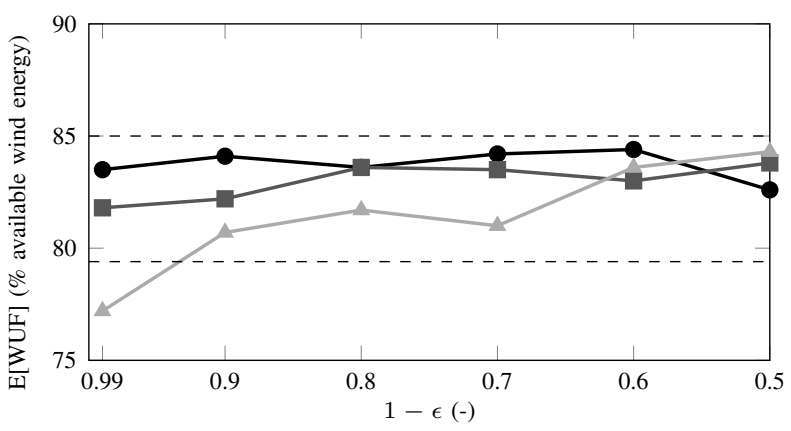

(b) Wind utilization factor (WUF)

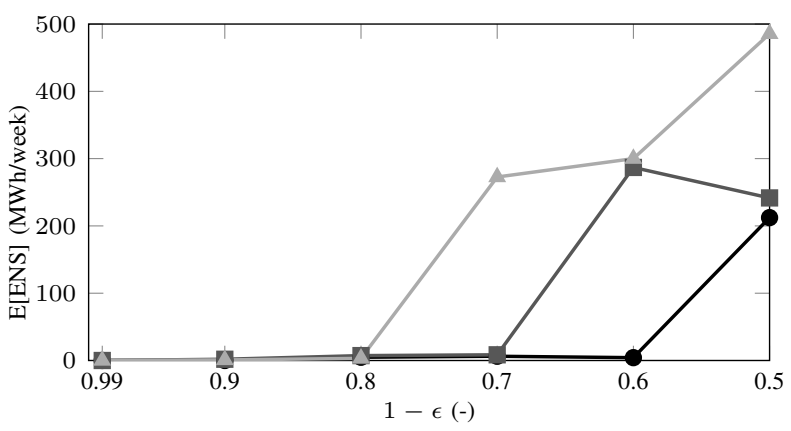

(c) Energy not served (ENS)

Fig. 4. The limited controllability of DR loads may lead to higher expected operating costs (TOC, Fig. 4a) due to less efficient UC schedules $(\epsilon \rightarrow 0$, too risk-averse SO), which results in lower wind utilization factors (WUF, Fig. $4 \mathrm{~b}$ ), or insufficient scheduled capacity to mitigate deviations from the optimal DR-load profile ( $\epsilon \rightarrow 0.5$, risk-neutral SO), which results in high energy not served-volumes (ENS, Fig. 4c). The total operating cost is normalized with respect to the corresponding operating cost when the DR load is not responsive. The dashed line at $90 \%$ indicates the operating cost attainable with perfectly controllable loads (arbitrage only). The solid lines consider the limited controllability of DR loads during the MC trials, the dashed lines do not.

total operating cost will - by definition, given our approach to the MC ED evaluation - be equal to that obtained assuming perfect controllability during UC scheduling (Fig. 4a).

However, such an approach leads to load shedding in case of unexpected DR load events (Fig. 4c), which increases the expected operating cost (Fig. 4a, solid lines). An optimal value of $\epsilon$, i.e. an optimal DR-risk policy, exists, formed by the tradeoff between the expected volume and cost of load shedding and the expected operating cost reduction associated with DR, and depends on the characterization of the controllability of the DR loads.

\section{CONCLUSION}

In order to quantify the operational effects associated with the introduction of residential demand response (DR) programs, we developed an integrated modeling approach. This model captures the effect of DR on the supply and demand side of an electric power system, and quantifies the attainable operating cost savings from a system perspective. Focusing on residential DR, we have applied this integrated model in a case study inspired by the Belgian power system, assuming a high wind energy penetration and a large-scale roll-out of DR-capable electric heating systems. Our numerical results show that DR-based arbitrage and regulation services could provide significant economic value. The observed operating cost savings amount to $6 \%$ on average for arbitrage and $7 \%$ for arbitrage and regulation combined. These operating cost savings may be attained without tampering with the thermal comfort of the residential consumers providing these services. If this thermal comfort can be violated, an additional operating cost reduction of $2 \%$ (arbitrage) to $3 \%$ (arbitrage and regulation) can be realized in this particular case study. However, the impact on the thermal comfort of the home owners would be significant and they may have to be compensated. Finally, we showed, using chance-constrained programming, that the controllability of the loads that participate in DR is critical to achieving these operating cost savings. Even with near perfect controllability, risk-averse SOs may see no value in DR. An optimal risk-policy exists and depends on the controllability of the DR loads.

However, the observed operating costs savings must be compared to the required investments in communication, transmission and distribution infrastructure to facilitate the participation of DR loads in the energy and reserve markets. Inspired by the analysis above, future work may focus on a detailed cost-benefit analysis for DR development. This analysis could be further elaborated by performing sensitivity analyses on parameters such as the load behavior, power system characteristics and representative time periods.

\section{ACKNOWLEDGMENT}

The computational resources and services used in this work were provided by the VSC (Flemish Supercomputer Center), funded by the Research Foundation - Flanders (FWO) and the Flemish Government department EWI. K. Bruninx is a postdoctoral research fellow of the Research Foundation - Flanders (FWO) and VITO, the Flemish Institute for Technological Research.

\section{REFERENCES}

[1] G. Strbac, "Demand side management: Benefits and challenges," Energy Policy, vol. 36, no. 12, pp. 4419-4426, 2008.

[2] D. S. Callaway, "Tapping the energy storage potential in electric loads to deliver load following and regulation, with application to wind energy," Energy Conversion and Management, vol. 50, no. 5, pp. 1389-1400, 2009.

[3] J. L. Mathieu, M. Kamgarpour, J. Lygeros, G. Andersson, and D. S. Callaway, "Arbitraging Intraday Wholesale Energy Market Prices With Aggregations of Thermostatic Loads," IEEE Transactions on Power Systems, vol. 30, no. 2, pp. 763-772, 2014.

[4] H. C. Gils, "Assessment of the theoretical demand response potential in europe," Energy, vol. 67, pp. 1-18, 2014. 
[5] D. Patteeuw, G. Reynders, K. Bruninx, C. Protopapadaki, E. Delarue, W. Dhaeseleer, D. Saelens, and L. Helsen, "CO2-abatement cost of residential heat pumps with active demand response: demand- and supply-side effects," Applied Energy, vol. 156, pp. 490-501, 2015.

[6] A. Arteconi, D. Patteeuw, K. Bruninx, E. Delarue, W. D'haeseleer, and L. Helsen, "Active demand response with electric heating systems : impact of market penetration," Applied Energy, vol. 177, pp. 636-648, 2016.

[7] H. Hao, B. M. Sanandaji, K. Poolla, and T. L. Vincent, "Aggregate Flexibility of Thermostatically Controlled Loads," IEEE Transactions on Power Systems, vol. 30, no. 1, pp. 189-198, 2015.

[8] B. Li and J. L. Mathieu, "Analytical Reformulation of ChanceConstrained Optimal Power Flow with Uncertain Load Control," in IEEE PowerTech, Eindhoven, The Netherlands, June 29 - July 2, 2015.

[9] Y. Zhang, S. Shen, and J. L. Mathieu, "Distributionally robust chanceconstrained optimal power flow with uncertain renewables and uncertain reserves provided by loads," IEEE Transactions on Power Systems, vol. 32, no. 2, pp. 1378-1388, 2017.

[10] N. O'Connell, P. Pinson, H. Madsen, and M. O'Malley, "Economic dispatch of demand response balancing through asymmetric block offers," IEEE Transactions on Power Systems, vol. 31, no. 4, pp. 2999-3007, 2016.

[11] D. Patteeuw, K. Bruninx, A. Arteconi, E. Delarue, W. D'haeseleer, and L. Helsen, "Integrated modeling of active demand response with electric heating systems coupled to thermal energy storage systems," Applied Energy, vol. 151, pp. 306-319, 2014.

[12] J. L. Mathieu, M. G. Vaya, and G. Andersson, "Uncertainty in the flexibility of aggregations of demand response resources," in IECON 2013. Vienna, Austria: IEEE, November 10-13, 2013, pp. 8052-8057.

[13] J. L. Mathieu, D. S. Callaway, and S. Kiliccote, "Variability in automated responses of commercial buildings and industrial facilities to dynamic electricity prices," Energy and Buildings, vol. 43, no. 12, pp. 3322-3330, 2011.

[14] N. Lu, D. P. Chassin, and S. E. Widergren, "Modeling uncertainties in aggregated thermostatically controlled loads using a state queueing model," IEEE Transactions on Power Systems, vol. 20, no. 2, pp. 725$733,2005$.

[15] K. Bruninx and E. Delarue, "Endogenous probabilistic reserve sizing and allocation in unit commitment models: cost-effective, reliable and fast," IEEE Transactions on Power Systems, vol. PP, no. 99, pp. 1-1, 2016.

[16] D. S. Callaway and I. A. Hiskens, "Achieving Controllability of Electric Loads," Proceedings of the IEEE, vol. 99, no. 1, pp. 184-199, 2011.

[17] A. Kosek, G. Costanzo, H. Bindner, and O. Gehrke, "An overview of demand side management control schemes for buildings in smart grids," in IEEE International Conference on Smart Energy Grid Engineering (SEGE), UOIT, Oshawa, Canada, August 28-30, 2013, pp. 1-9.

[18] B. Shen, G. Ghatikar, Z. Lei, J. Li, G. Wikler, and P. Martin, "The role of regulatory reforms, market changes, and technology development to make demand response a viable resource in meeting energy challenges," Applied Energy, vol. 130, pp. 814 - 823, 2014.

[19] D. Bienstock, M. Chertkov, and S. Harnett, "Chance-Constrained Optimal Power Flow: Risk-Aware Network Control under Uncertainty," SIAM Review, vol. 56, no. 3, pp. 461-495, 2014.

[20] M. Lubin, Y. Dvorkin, and S. Backhaus, "A Robust Approach to Chance Constrained Optimal Power Flow with Renewable Generation," IEEE Transactions on Power Systems, vol. 31, no. 5, pp. 1-11, 2015.

[21] K. Bruninx, Y. Dvorkin, E. Delarue, H. Pandžić, W. D'haeseleer, and D. S. Kirschen, "Coupling Pumped Hydro Energy Storage with Unit Commitment," IEEE Transactions on Sustainable Energy, vol. 7, no. 2, pp. 786-796, 2016.

[22] D. Patteeuw and L. Helsen, "Residential buildings with heat pumps, a verified bottom-up model for demand side management studies," in 9th International Conference on System Simulation in Buildings, no. 1, Liège, Belgium, December 10-12, 2014, pp. 1-19.

[23] G. Reynders, J. Diriken, and D. Saelens, "Quality of grey-box models and identified parameters as function of the accuracy of input and observation signals," Energy and Buildings, vol. 82, pp. 263-274, 2014

[24] N. Good, E. Karangelos, A. Navarro-Espinosa, and P. Mancarella, "Optimization Under Uncertainty of Thermal Storage-Based Flexible Demand Response With Quantification of Residential User's Discomfort," IEEE Transactions on Smart Grid, vol. 6, no. 5, pp. 2333-2342, 2015.

[25] D. Patteeuw, G. P. Henze, and L. Helsen, "Comparison of load shifting incentives for low-energy buildings with heat pumps to attain grid flexibility benefits," Applied Energy, vol. 167, pp. 80-92, 2016.

[26] K. Bruninx, "Improved modeling of unit commitment decisions under uncertainty," $\mathrm{PhD}$ dissertation, KU Leuven, 2016.
[27] M. Vrakopoulou, K. Margellos, J. Lygeros, and G. Andersson, "A probabilistic framework for reserve scheduling and n-1 security assessment of systems with high wind power penetration," IEEE Transactions on Power Systems, vol. 28, no. 4, pp. 3885-3896, 2013.

[28] K. Margellos, P. Goulart, and J. Lygeros, "On the road between robust optimization and the scenario approach for chance constrained optimization problems," IEEE Transactions on Automatic Control, vol. 59, no. 8, pp. 2258-2263, 2014

[29] R. Baetens and D. Saelens, "Modelling uncertainty in district energy simulations by stochastic residential occupant behaviour," Journal of Building Performance Simulation, vol. 1493, pp. 1-17, 2015.

[30] P. Pinson, H. Madsen, H. A. Nielsen, G. Papaefthymiou, and B. Klöck1, "From probabilistic forecasts to statistical scenarios of short-term wind power production," Wind Energy, vol. 12, no. 1, pp. 51-62, 2009.

Kenneth Bruninx (S'15-M'16) received the M.Sc. degree in energy engineering in 2011 and the Ph.D. degree in mechanical engineering in 2016, both from the Univeristy of Leuven (KU Leuven), Belgium.

Currently, he is working as a post-doctoral research fellow of the Research Foundation - Flanders (FWO) at (i) the University of Leuven Energy Institute, TME branch (energy conversion), (ii) VITO (the Flemish Institute for Technological Research) and (iii) EnergyVille, a joint venture of KU Leuven, VITO and IMEC.

Yury Dvorkin (S'11-M'16) received his Ph.D. degree from the University of Washington, Seattle, WA, USA, in 2016.

Dvorkin is currently an Assistant Professor in the Department of Electrical and Computer Engineering at New York University, New York, NY, USA. Dvorkin was awarded the 2016 Scientific Achievement Award by Clean Energy Institute (University of Washington) for his doctoral dissertation Operations and Planning in Sustainable Power Systems. His research interests include short- and long-term planning in power systems with renewable generation and power system economics.

Erik Delarue (M'15) received the M.Sc. degree in mechanical engineering in 2005 and the Ph.D. degree in mechanical engineering in 2009, both from the University of Leuven (KU Leuven), Belgium.

Currently, he is an Assistant Professor at the University of Leuven Energy Institute, TME branch (energy conversion).

William D'haeseleer (M'15) received his M.S. degree in Electro-Mechanical Engineering and a Master in Nuclear Engineering from the University of Leuven (KU Leuven), Belgium, in 1980 and 1982, respectively. He obtained another M.S. degree in Electrical Engineering and the Ph.D. degree from the University of Wisconsin-Madison, USA, in 1983 and 1988, respectively. Currently, he is a full-time Professor in the College of Engineering of the KU Leuven and Director of the University of Leuven Energy Institute.

Daniel S. Kirschen (M'86SM'91F'07) received the electrical and mechanical engineer's degrees from the Universit Libre de Bruxelles, Belgium, in 1979, and the M.Sc. and Ph.D. degrees from the University of Wisconsin, Madison, WI, USA, in 1980 and 1985, respectively. He is currently Close Professor of Electrical Engineering at the University of Washington, Seattle, WA, USA. His research focuses on smart grids, the integration of renewable energy sources in the grid, power system economics and power system security. 